Cite this: Soft Matter, 2014, 10, 3938

\title{
Viscoelasticity of nematic liquid crystals at a glance
}

\author{
Fabio Giavazzi, ${ }^{\text {*a }}$ Stefano Crotti, ${ }^{a}$ Antonio Speciale, ${ }^{a}$ Francesca Serra, ${ }^{a}$ \\ Giuliano Zanchetta, ${ }^{a}$ Veronique Trappe, ${ }^{b}$ Marco Buscaglia, ${ }^{a}$ Tommaso Bellini ${ }^{a}$ \\ and Roberto Cerbino*a
}

\begin{abstract}
Polarised microscopy is shown to be a powerful alternative to light scattering for the determination of the viscoelasticity of aligned nematic liquid crystals. We perform experiments in a wide range of temperatures by using an adapted version of the recently introduced differential dynamic microscopy technique, which enables us to extract scattering information directly from the microscope images. A dynamic analysis of the images acquired in different geometries provides the splay, twist and bend viscoelastic ratios. A static analysis allows a successful determination of the bend elastic constant. All our results are in excellent agreement with those obtained with the far more time-consuming depolarised light scattering techniques. Remarkably, a noteworthy extension of the investigated temperature-range is observed, owing to the lower sensitivity of microscopy to multiple scattered light. Moreover, we show that the unique space-resolving capacities of our method enable us to investigate nematics in the presence of spatial disorder, where traditional light scattering fails. Our findings demonstrate that the proposed scattering-with-images approach provides a space-resolved probe of the local sample properties, applicable also to other optically anisotropic soft materials.
\end{abstract}

Received 19th January 2014

Accepted 3rd March 2014

DOI: $10.1039 / c 4 s m 00145 a$

www.rsc.org/softmatter suitable scattering geometries are used. ${ }^{5,6}$ Small-angle DDLS measurements are quite demanding, requiring very clean sample cells and optical surfaces to minimise the unwanted scattered light (stray light). In addition, measurements at several scattering angles are often necessary, which increases substantially the overall measurement time to achieve the sample characterisation. Another possible - although scantily explored - route for the characterisation of the LC viscoelasticity exploits the fact that the director fluctuations can be easily visualised in real space by means of depolarised microscopy and recorded with a pixel detector (camera) for subsequent analysis. This idea was originally used in ref. 7, where spatial Fourier transforms of microscope images of nematic LC were analysed in time to extract the twist viscoelastic ratio. While the latter was found to be in agreement with previous DDLS measurements, no information about the bend and splay viscoelastic rations could be retrieved, leaving the full potential of dynamic microscopy experiments still unexpressed. A particularly promising technique for the full characterisation of the LC viscoelasticity is represented by the recently introduced Differential Dynamic Microscopy (DDM). ${ }^{8}$ In its simplest implementation, DDM allows performing Dynamic Light Scattering (DLS) experiments with a camera-equipped microscope, by recording a short movie of the sample and processing it through a combination of subtraction of images acquired at different times and a spatial Fourier analysis. The sample dynamics is then recovered by looking at the increase of the square amplitude of each Fourier mode as the time separation between the two subtracted images is made larger, which 
provides the intermediate scattering function for the corresponding wave vector. ${ }^{9}$ This approach has been successfully demonstrated with a variety of samples including colloids and bacteria, both in bright field, ${ }^{8-10}$ phase contrast, ${ }^{11-13}$ and fluorescence wide-field ${ }^{14}$ or confocal ${ }^{15}$ microscopy. We shall prove here that DDM in combination with properly oriented polarisers - hereinafter named polarised differential dynamic microscopy or pDDM - allows performing DDLS experiments with a microscope and permits the full characterisation of LC viscoelastic ratios in nematics. To this aim we first develop a theoretical description of dynamic microscopy experiments with optically anisotropic samples. We then succeed in measuring experimentally the three viscoelastic ratios in a nematic LC sample with suitable alignment, thereby demonstrating PDDM as a powerful tool for the rapid characterisation of nematics. The use of pDDM for the extraction of the elastic constants necessitates alignment-dependent theoretical expressions describing the effect of the light propagation in a distorted medium on the image intensity. Even though deriving such expressions is beyond the aim of this work, we adapt recent results developed in ref. 16, primarily for the description of electroconvection patterns, for one of the experimental geometries used in this study. As a result we could extract the bend elastic constant at various temperatures and find it to be in excellent agreement with literature data. We also show that our imaging-based approach allows extracting the viscoelastic parameters in a heterogeneous planar nematic, by means of a space-resolved experiment that would be practically impossible with DDLS. Our results suggest a routine use of microscopes for the determination of the viscoelastic properties of various optically anisotropic fluids, such as for instance lyotropic liquid crystals made of anisotropic macromolecules or colloids. ${ }^{17-20}$

\section{Nematodynamics}

The relaxation of orientational fluctuations of the director is usually described in the framework of nematodynamics theory. ${ }^{5,21}$ The elastic cost of deformation of the nematic order is expressed by the free-energy volume density

$$
f=\frac{1}{2}\left\{K_{11}(\nabla \cdot \hat{n})^{2}+K_{22}[\hat{n} \cdot(\nabla \times \hat{n})]^{2}+K_{33}[\hat{n} \times(\nabla \times \hat{n})]^{2}\right\}
$$

where the unit vector $\hat{n}(\vec{r})=\hat{n}_{0}+\delta \vec{n}(\vec{r})$ provides the local orientation of the director. In fact, eqn (1) defines the three elastic constants $K_{11}, K_{22}$ and $K_{33}$ that are associated with splay, twist and bend deformations, respectively.

Nematodynamics uses eqn (1) as a starting point to write equations that account for conservation of the linear and angular momentum, suitably complemented by the constitutive equations for the material. ${ }^{5,21}$ Exact solutions of such equations can be obtained by their linearization for small fluctuations of velocity $(\delta \vec{v})$ and direction $(\delta \vec{n})$ around the equilibrium solution $\left(\vec{v}=0\right.$ and $\left.\hat{n}=\hat{n}_{0}\right)$. By introducing the Fourier transform $\delta \vec{n}(\vec{Q})=$ $\int \delta \vec{n}(\vec{r}) \exp (-\mathrm{i} \vec{Q} \cdot \vec{r}) \mathrm{d} \vec{r}$ and by choosing an orthonormal reference system $\left(\hat{e}_{1}, \hat{e}_{2}, \hat{e}_{3}=\hat{n}_{0}\right)$, such that for each wave vector $\vec{Q}$ the unit vector $\hat{e}_{2}$ is perpendicular to $\vec{Q}$ and $\hat{e}_{1}$ is perpendicular to $\hat{e}_{2}$, the free energy density in eqn (1) assumes the particularly useful diagonal form

$$
F=\frac{1}{2 V} \sum_{Q} \sum_{\nu=1,2}\left|\delta n_{\nu}(\vec{Q})\right|^{2}\left(K_{v v} Q_{\perp}^{2}+K_{33} Q_{\|}{ }^{2}\right)
$$

in the reciprocal space. For each $\vec{Q}=\vec{Q}_{\|}+\vec{Q}_{\perp}=Q_{\|} \hat{n}_{0}+Q_{\perp} \hat{e}_{1}$ fluctuations of the nematic director are accordingly decomposed in two collective normal modes $(v=1,2)$, defined by the relative orientation of $\hat{n}_{0}$ and $\vec{Q}$, and schematically depicted in Fig. 1 . Mode 1 describes director fluctuations $\delta \vec{n}$ perpendicular to $\hat{n}_{0}$ in the $\left(\hat{n}_{0}, \vec{Q}\right)$ plane, which are due to splay and bend deformations. Fluctuations perpendicular to the $\left(\hat{n}_{0}, \vec{Q}\right)$ plane define mode 2, which is a combination of twist and bend deformations. Relaxation of the modes occurs exponentially with a rate

$$
\Gamma_{\nu}(\vec{Q})=\frac{K_{\nu v} Q_{\perp}^{2}+K_{33} Q_{\|}^{2}}{\eta_{\nu}(\vec{Q})}
$$

where $\eta_{\nu}(\vec{Q})$ denotes two $Q$-dependent viscosities defined in terms of the Leslie viscosities ${ }^{5} \alpha_{i}(i=1,2,3,4,5,6)$ as

$$
\eta_{1}(\vec{Q})=\gamma_{1}-\frac{\left(Q_{\perp}{ }^{2} \alpha_{3}-Q_{\|}{ }^{2} \alpha_{2}\right)^{2}}{Q_{\perp}{ }^{4} \eta_{b}+Q_{\perp}{ }^{2} Q_{\|}{ }^{2}\left(\alpha_{1}+\alpha_{3}+\alpha_{4}+\alpha_{5}\right)+Q_{\|}{ }^{4} \eta_{c}}
$$

and

$$
\eta_{2}(\vec{Q})=\gamma_{1}-\frac{\alpha_{2}^{2} Q_{\|}^{2}}{Q_{\perp}{ }^{2} \eta_{a}+Q_{\|}^{2} \eta_{c}}
$$

where $\gamma_{1}=\alpha_{3}-\alpha_{2}, \eta_{\alpha}=\alpha_{4} / 2, \eta_{b}=\left(\alpha_{2}+\alpha_{4}+\alpha_{6}\right) / 2$, and $\eta_{c}=\left(-\alpha_{2}+\alpha_{4}+\alpha_{5}\right) / 2$.

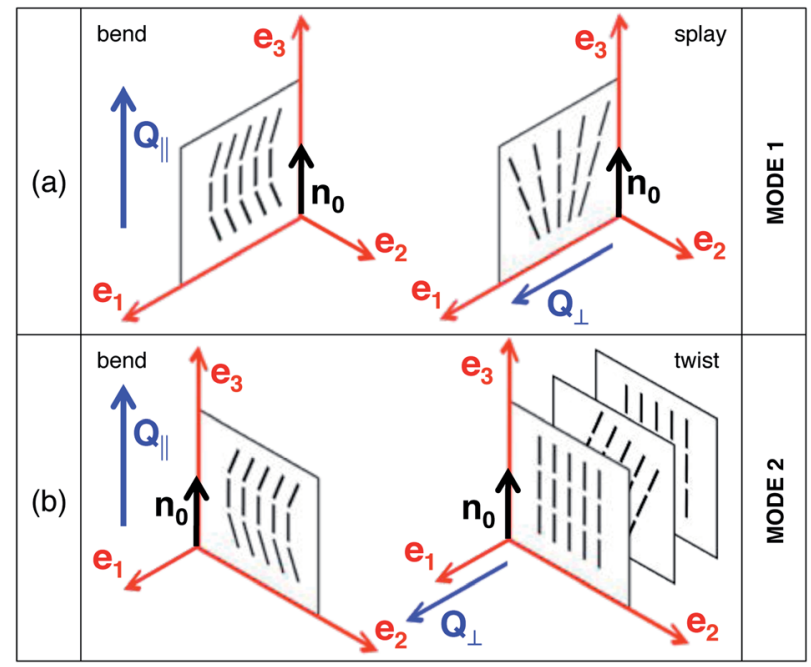

Fig. 1 Cartesian reference system $\left(\hat{e}_{1}, \hat{e}_{2}, \hat{e}_{3}=\hat{n}_{0}\right)$ used to decompose the director fluctuations in mode 1 and mode 2. The wave vector $\vec{Q}$ of the fluctuations (not shown) and the director $\hat{n}_{0}$ form a plane. The unit vector $\hat{e}_{2}$ is perpendicular to that plane. With this choice $\vec{Q}$ lies in the $\left(\hat{n}_{0}, \hat{e}_{1}\right)$ plane and can be seen as the sum of $\vec{Q}_{\|}$and $\vec{Q}_{\perp}$. (a) Mode 1 corresponds to bend and splay distortions of the director. (b) Mode 2 accounts for bend and twist. The vector $\delta \vec{n}(\vec{r})$ (not shown) is oriented along $\hat{e}_{1}$ for mode 1 (a) and along $\hat{e}_{2}$ for mode 2 (b). 
These rather complex expressions can be simplified in some limit cases. For the cases of interest in the present work, one has:

$$
\begin{gathered}
\eta_{1}(\vec{Q}) \rightarrow\left\{\begin{array}{ccc}
\eta_{\text {splay }} \doteq & \gamma_{1}-\frac{\alpha_{3}^{2}}{\eta_{b}}, & Q_{\perp} \gg Q_{\|} \\
\eta_{\text {bend }} \doteq & \gamma_{1}-\frac{\alpha_{2}^{2}}{\eta_{c}}, & Q_{\perp} \ll Q_{\|}
\end{array}\right. \\
\eta_{2}(\vec{Q}) \rightarrow\left\{\begin{array}{ccc}
\eta_{\text {twist }} \doteq & \gamma_{1}, & Q_{\perp} \gg Q_{\|} \\
\eta_{\text {bend }} \doteq & \gamma_{1}-\frac{\alpha_{2}^{2}}{\eta_{c}}, & Q_{\perp} \ll Q_{\|}
\end{array}\right.
\end{gathered}
$$

which define $\eta_{\text {splay }}, \eta_{\text {twist }}, \eta_{\text {bend }}$, and in turn result in the following expressions for the relaxation rates of the two modes:

$$
\begin{gathered}
\Gamma_{1}(\vec{Q}) \rightarrow \begin{cases}\Gamma_{\text {splay }} \doteq \frac{K_{11}}{\eta_{\text {splay }}} Q_{\perp}{ }^{2}, & Q_{\perp} \gg Q_{\|} \\
\Gamma_{\text {bend }} \doteq \frac{K_{33}}{\eta_{\text {bend }}} Q_{\|}{ }^{2}, & Q_{\perp} \ll Q_{\|}\end{cases} \\
\Gamma_{2}(\vec{Q}) \rightarrow\left\{\begin{array}{lll}
\Gamma_{\text {twist }} \doteq & \frac{K_{22}}{\eta_{\text {twist }}} Q_{\perp}{ }^{2}, & Q_{\perp} \gg Q_{\|} \\
\Gamma_{\text {bend }} \doteq & \frac{K_{33}}{\eta_{\text {bend }}} Q_{\|}{ }^{2}, & Q_{\perp} \ll Q_{\|}
\end{array}\right.
\end{gathered}
$$

For each of the three deformations, the viscoelastic ratio is thus in the form $K / \eta$ where $K$ is a deformation-dependent elastic constant (measured in newton) and $\eta$ is the corresponding viscosity (measured in Pa s). In the next paragraph we will show how it is possible to exploit the limits in eqn (8) and (9) in DDM experiments.

\section{Dynamic microscopy of fluctuating nematics}

In this paragraph we first briefly recall the working principles of bright-field DDM, as introduced in ref. 8 and 9. In addition, we describe DDM in the presence of polarising elements, which are the essence of the pDDM method. Our description includes the outline of novel experimental geometries that are used here for the characterisation of the viscoelasticity of nematics.

\section{Bright-field DDM}

DDM is a near-field (or deep-Fresnel) scattering technique ${ }^{22}$ that allows the recovery of scattering information about the sample by analysing sequences of images (movies) acquired close to the sample (deep-Fresnel regime) rather than in the sample far-field (Fig. 2). The main idea of DDM is to extract from microscope images a signal that is proportional to the density fluctuations within the sample. In this way, by means of Fourier transform analysis, it is possible to quantify relevant statistical quantities such as the static and the dynamic structure factor, for comparison with suitable theoretical models or with analogous quantities extracted from far-field scattering experiments. ${ }^{9}$

In bright-field DDM experiments such a task is easily tackled for weakly scattering samples i.e. whenever the intensity of the

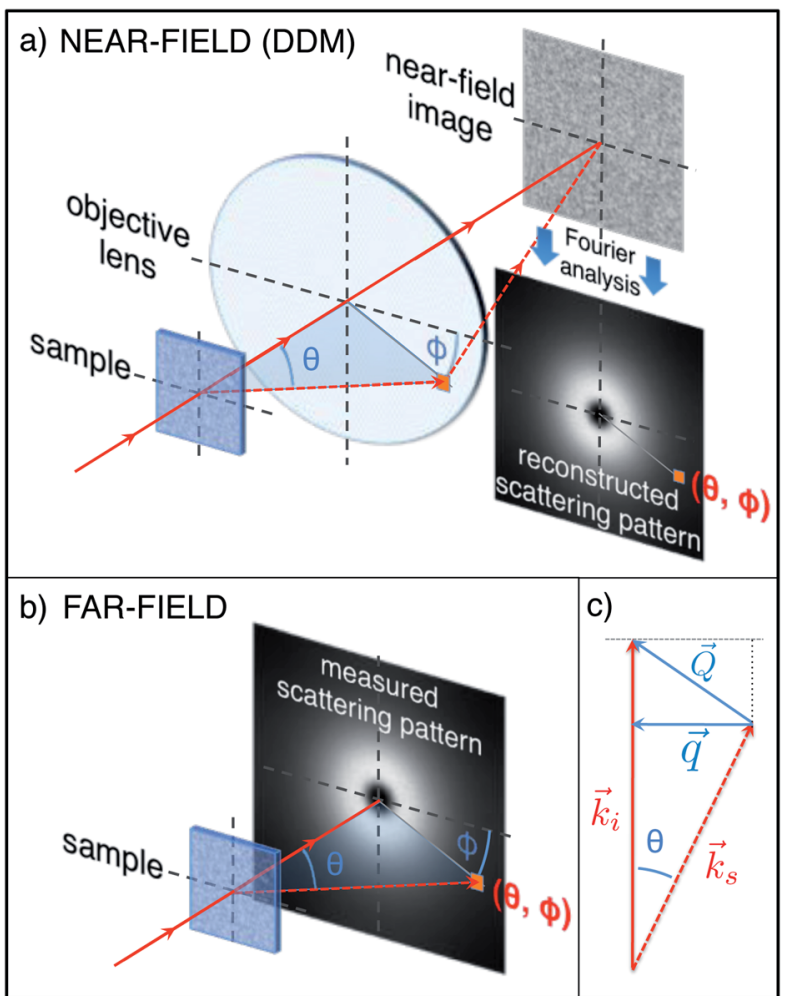

Fig. 2 In a DDM experiment (a) light impinging on the sample is scattered at various angles and is collected by the objective lens. Twodimensional microscope images of the sample are Fourier analysed and information equivalent to a traditional far-field scattering experiment (b) is recovered. A generic scattered ray (wave propagating) with polar angle $\theta$ and azimuthal angle $\phi$ (dashed line), which corresponds to the point $(\theta, \phi)$ in the far-field scattering pattern (b), is collected by the lens in a DDM experiment (a) and contributes to the images. The contribution of each scattered ray (wave) can be isolated by means of a two dimensional Fourier analysis, which is based on the two-dimensional projection $\vec{q}$ (defined in eqn (12)) of the wave vector $\vec{Q}$ transferred during the scattering process (c). The length $k_{\mathrm{s}}$ of the scattered wave vector $\vec{k}_{\mathrm{s}}$ may differ in general from the length $k_{\mathrm{i}}$ of the incident wave vector $\vec{k}_{\mathrm{i}}$ (inelastic scattering).

transmitted beam $I_{0}=E_{0}^{*} E_{0}$ is way larger than the intensity of the scattered light $I_{\mathrm{s}}=E_{\mathrm{s}}^{*} E_{\mathrm{s}}$, where $E_{0}$ and $E_{\mathrm{s}}$ are the incident and scattered fields, respectively. Indeed, fulfillment of the heterodyne condition $I_{\mathrm{S}} \ll I_{0}$ guarantees that the intensity of each microscope image can be written as

$$
I(x, y, t)=\left|E_{0}+E_{\mathrm{s}}(x, y, t)\right|^{2} \simeq I_{0}+2 \operatorname{Re}\left[E_{0}^{*} E_{\mathrm{s}}(t)\right]
$$

where the homodyne term $I_{\mathrm{s}}$ has been neglected and where Re [...] is the real part of the argument. Eqn (10) shows that a measure of the intensity $I(x, y, t)$ enables one to access the real part of the fluctuating scattering field $E_{\mathrm{s}}(x, y, t)$, a consequence of the intrinsic interferometric nature of the method. This fact can be exploited if an effective procedure for removing the transmitted light intensity $I_{0}$ is found. Among the possible choices, ${ }^{9}$ a very common one is to calculate the algebraic difference between two images acquired at different times $t_{0}$ and $t_{0}+\Delta t$ to obtain the difference image 


$$
\begin{aligned}
d\left(x, y, t_{0}, \Delta t\right) & \doteq I\left(x, y, t_{0}+\Delta t\right)-I\left(x, y, t_{0}\right) \\
& \simeq 2 \operatorname{Re}\left\{E_{0}^{*}\left[E_{\mathrm{s}}\left(t_{0}+\Delta t\right)-E_{\mathrm{s}}\left(t_{0}\right)\right]\right\}
\end{aligned}
$$

For stationary ergodic samples the dependence on the reference time $t_{0}$ can be neglected since all the images obtained by subtracting pairs separated in time by the same value of $\Delta t$ are statistically equivalent. This allows averaging of the spatial Fourier power spectra of all difference images with the same $\Delta t$ to obtain the image structure function

$D\left(q_{x}, q_{y}, \Delta t\right)=\left\langle\left|\int \mathrm{d} q_{x} \mathrm{~d} q_{x} \exp \left[-\mathrm{i}\left(x q_{x}+y q_{y}\right) d\left(x, y, t_{0}, \Delta t\right)\right]\right|^{2}\right\rangle_{t_{0}}$.

The image structure function is studied for each $\vec{q}$ as a function of $\Delta t$ by fitting the trend of the experimental data points with the theoretical expression

$$
D(\vec{q}, \Delta t)=A(\vec{q})[1-g(\vec{q}, \Delta t)]+B(\vec{q})
$$

where $B(\vec{q})$ is a background term that accounts for the noise of the detection chain, $A(\vec{q})=T(\vec{q}) I(\vec{q}), I(\vec{q})$ is the intensity scattered by the sample and $T(\vec{q})$ is a transfer function that depends on the microscope. ${ }^{9}$ It is particularly relevant that $g(\vec{q}, \Delta t)$, the socalled intermediate scattering function, is the quantity normally accessible in DLS experiments and this provides the link between DDM and DLS. ${ }^{9}$ For most of the systems previously analysed with $\mathrm{DDM}^{\mathbf{8 - 1 2 , 1 4 , 1 5}}$ the image structure function bore a circular symmetry (like in Fig. $2 \mathrm{a}$ and b), such that an azimuthal average for $q=\sqrt{q_{x}^{2}+q_{y}^{2}}$ was performed. The structure function $D(q, \Delta t)$ was thus typically studied as a function of $\Delta t$ by fitting the trend of the experimental data points with the theoretical expression

$$
D(q, \Delta t)=A(q)[1-g(q, \Delta t)]+B(q) .
$$

It is worth noting that the wave vector $\vec{q}$ accessible in DDM experiments is a two-dimensional projection of the wave vector $\vec{Q}=\vec{k}_{\mathrm{i}}-\vec{k}_{\mathrm{s}}$ transferred during a scattering process, where $\vec{k}_{\mathrm{i}}$ and $\vec{k}_{\mathrm{s}}$ are the incident and the scattered wave vector, respectively (see Fig. 2c). As a consequence of the small wave vectors accessible in DDM experiments, typically well below $10 \mu \mathrm{m}^{-1}$, the difference between $\vec{q}$ and $\vec{Q}$ is usually negligible, as is a common practice for camera-based far-field small angle elastic scattering experiments. ${ }^{\mathbf{2 3 2 4}}$

\section{DDM with polarising elements (pDDM)}

Orientational fluctuations of the nematic director can change the polarisation state of incident light. The two modes decomposing the fluctuations of the nematic director introduced in eqn (2) can be easily probed in a scattering experiment, where a nematic liquid crystal slab is illuminated by a plane wave with wave vector $\vec{k}_{\mathrm{i}}$ and polarisation direction $\hat{i}$. The scattered light is then collected in a direction specified by the wave vector $\vec{k}_{\mathrm{f}}$ and the polarisation direction $\hat{f}$. The polarisation directions $\hat{i}$ and $\hat{f}$ are usually selected by use of linear polarising elements placed before and after the LC sample, respectively. The scattering differential cross-section $\sigma_{\mathrm{d}}$ in such experiments is given by

$$
\sigma_{\mathrm{d}}(\vec{Q})=\text { const. } \sum_{\nu=1,2} \frac{\left(i_{\nu} f_{z}+i_{z} f_{\nu}\right)^{2}}{K_{\nu \nu} Q_{\perp}{ }^{2}+K_{33} Q_{\|}{ }^{2}}
$$

where $i_{\nu}=\hat{i} \cdot \hat{e}_{\nu}, i_{\mathrm{z}}=\hat{i} \cdot \hat{n}_{0}, f_{\nu}=\hat{f} \cdot \hat{e}_{\nu}, f_{z}=\hat{f} \cdot \hat{n}_{0}$. The dynamics is described by the intermediate scattering function, which is given by

$$
g(\vec{Q}, \Delta t)=g_{1}(\vec{Q}) \exp \left[-\Gamma_{1}(\vec{Q}) \Delta t\right]+g_{2}(\vec{Q}) \exp \left[-\Gamma_{2}(\vec{Q}) \Delta t\right]
$$

where the relaxation rates $\Gamma_{\nu}(\vec{Q})$ have been already defined in eqn (3) and where $g_{1}(\vec{Q})+g_{2}(\vec{Q})=1$, with $g_{1}(\vec{Q})$ and $g_{2}(\vec{Q})$ depending on $\sigma_{\mathrm{d}}(\vec{Q})$. The presence of the term $\left(i_{v} f_{z}+i_{z} f_{v}\right)^{2}$ in eqn (15) shows that the relative contribution of each mode to the scattered light can be suppressed by a careful choice of the orientation of the director and of the polarising elements. As recently reviewed in ref. 6 , several far field scattering geometries have been proposed and reported in the literature to take advantage of this possibility. One of the main advantages of pDDM is that we can exploit known results from light scattering theory and we do not need ad hoc calculations, at least if we want to extract dynamic information. In principle, the investigation with DDM of the depolarised scattering of light from orientational fluctuations of the director requires thus a simple strategy: equipping the microscope with two linear polarising elements and performing a DDM analysis without resorting to an azimuthal averaging of the image structure function in the Fourier space. However, the ostensible simplicity of the experimental strategy can be misleading. Indeed, validity of eqn (10) is based on the assumption that the transmission of light through the sample remains large enough that the homodyne term $I_{\mathrm{s}}=E_{\mathrm{s}}^{*} E_{\mathrm{s}}$ can be safely neglected. An immediate consequence of such requirement is that typical geometries employed in DDLS experiments, where the sample is sandwiched between crossed polarisers and the signal of interest is a small intensity contribution superimposed to an ideally zero background, cannot be used directly with DDM. Indeed, under these conditions the homodyne term is dominant, eqn (10) becomes a bad approximation and the easy connection between DDM and farfield scattering experiments is lost. However, this difficulty can be overcome by identifying experimental geometries with orientation of the director and of the polarising elements that allow for the presence of a sufficiently intense transmitted beam. This problem is somehow similar to the one encountered in ref. 25, where the dynamics of randomly oriented colloidal particles was studied with near-field scattering. However, in the present case, it is possible to take advantage of well established procedures to fix the alignment of the director at the cell surfaces, as sketched in Fig. 3.

We have thus decided to first study LC samples with homeotropic and (homogeneous) planar alignment of the director at the cell surfaces (Fig $3 \mathrm{a}_{1}$ and $\mathrm{a}_{2}$ ), which represent two cases often encountered in the literature. The general scheme of our pDDM experiments is sketched in Fig. 3 where we describe the common features of all the experiments that we have conducted. The specific features of each experimental geometry can be instead appreciated by inspecting Fig. 4-7, where we have dropped the objective lens and the microscope image 


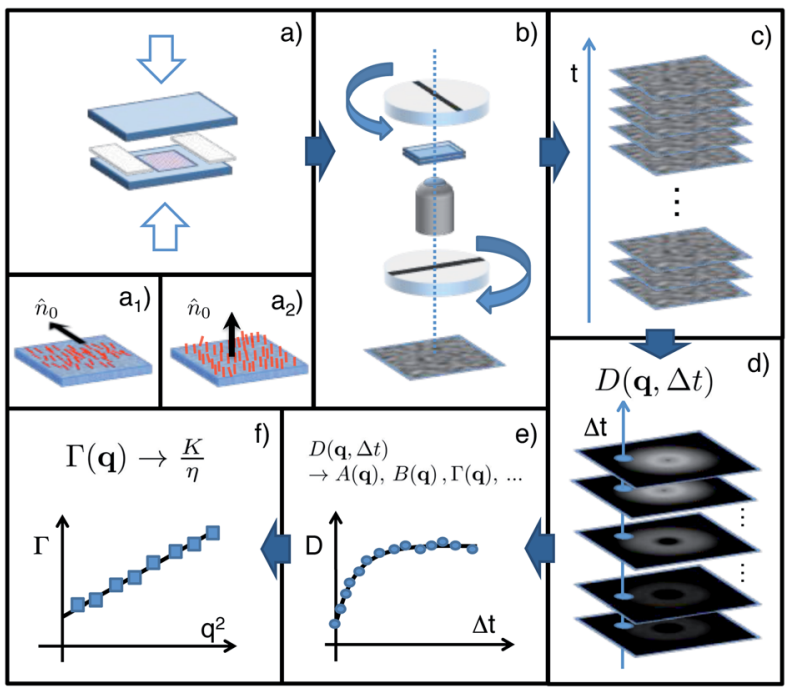

Fig. 3 Sketch of the pDDM experimental and data analysis procedure. (a) The sample is confined between two glass slides whose surfaces are treated in order to promote the planar $\left(a_{1}\right)$ or the homeotropic $\left(a_{2}\right)$ alignment of the director. (b) The sample cell is positioned on the microscope stage between two polarising elements. The polarisers are mutually oriented according to the modes to be probed (see text). (c) A stack of digital images of the sample is acquired with a fixed frame rate. (d) For each $\Delta t$, the 2D image structure function $D(\vec{q}, \Delta t)$ is calculated by averaging the Fourier power spectrum of the difference of images separated in time by the same time delay $\Delta t$. (e) The fit of $D(\vec{q}, \Delta t)$ as a function of $\Delta t$ allows the estimation of the $q$-dependent amplitudes $A$, the rates $\Gamma$ and the camera noise B (see eqn (13) and (16)). (f) The linear fit of each $\Gamma$ as a function of $q^{2}$ in selected geometry-dependent directions allows the estimation of the corresponding viscoelastic ratio, according to eqn (3).

to focus the attention on the reconstructed scattering pattern $A\left(q_{x}, q_{y}\right)=D\left(q_{x}, q_{y}, \Delta t \rightarrow \infty\right)$ and its symmetries. For all these geometries we will also specify the correct relations between the amplitude $Q$ of the three-dimensional wave vector of the fluctuations and the amplitude $q$ of the two-dimensional wave vector associated with the image Fourier transform. We note that light scattering from liquid crystals is in general inelastic $\left(k_{\mathrm{s}} \neq k_{\mathrm{i}}\right)$ with $\Delta q=\left|k_{\mathrm{i}}-k_{\mathrm{s}}\right|_{\max }=k_{0} \Delta m$, where $\Delta m=\left|m_{\mathrm{e}}-m_{\mathrm{o}}\right|$ is the difference between the extraordinary and ordinary refractive indices of the sample and $k_{0}$ is the incident wave vector in the vacuum.

Planar alignment - geometry P1. In this geometry the polariser and the analyser are crossed and the director forms an angle $\pi / 8$ with the polariser (Fig. 4). This choice for the angle guarantees not only a fairly intense transmitted beam intensity but also the linearity between the change in intensity and the (small) local orientational fluctuation of the director. As customarily done in optics, the linearly polarised incident light can be decomposed into two components, one with the polarisation direction of the electric field perpendicular to the director (ordinary light) and the other one parallel to it (extraordinary light). A similar decomposition can be made for the scattered light where both the elastic and inelastic scattering processes contribute to the scattering pattern. However, if the scattering pattern is analysed in the two directions (bow-

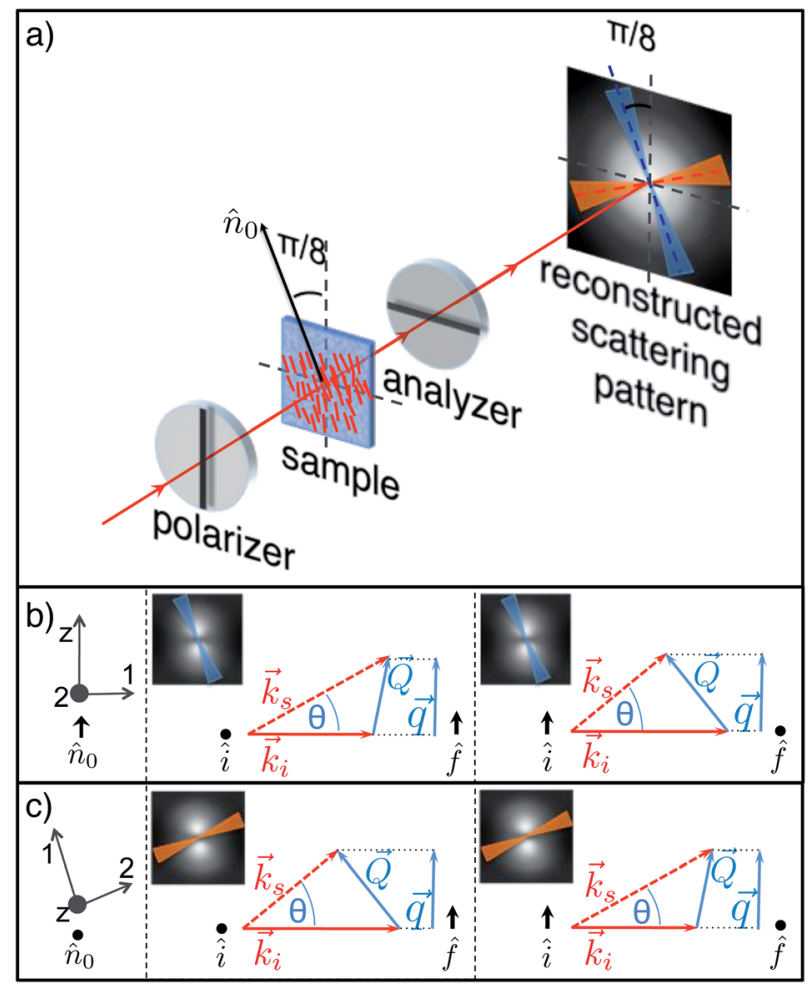

Fig. 4 (a) Sketch of a pDDM experiment in the P1 geometry. Unpolarised light crosses a linearly polarising element (polariser) and interacts with the nematic sample, whose director is placed at $\pi / 8$ with respect to the polariser axis. Both the transmitted beam and the scattered light encounter a second polarising element (analyser), perpendicular to the first one. Microscope images (not shown) are acquired and processed as described in the text to recover information equivalent to a traditional far-field scattering experiment. If the scattering pattern is analysed along the direction parallel (blue online) or perpendicular (red online) to the director, the contribution of polarised scattering is negligible. (b) For $\vec{q} \| \hat{n}_{0}$ (corresponding reference system on the left) the two combinations of interest are: incident ordinary light and scattered extraordinary light (centre); incident extraordinary light and scattered ordinary light (right). (c) For $\vec{q} \perp \hat{n}_{0}$ (corresponding reference system on the left) the two combinations of interest are: incident extraordinary light and scattered ordinary light (centre); incident ordinary light and scattered extraordinary light (right).

ties in Fig. 4a) that are parallel (centre in Fig. $4 \mathrm{~b}$ and c) and perpendicular (right in Fig. 4b and c) to the director, the contribution of polarised scattering is negligible. The use of eqn (15) with the proper reference system allows the estimation of the contribution of the two modes by recalling that the scattering of each mode is proportional to $\left(i_{v} f_{z}+i_{z} f_{\mathrm{v}}\right)^{2}$, with $v=1,2$.

- for director orientation modulations with wave vector $\vec{q}$ parallel to $\hat{n}_{0}$ the suitable reference system is drawn on the left side of panel b in Fig. 4. In this direction, only mode 2 can be thus probed. Indeed, for ordinary incident light and extraordinary scattered light (Fig. 4 , panel b, centre) one has $i_{1}=$ $i_{z}=f_{1}=f_{2}=0$, which implies $\left(i_{1} f_{z}+i_{2} f_{1}\right)^{2}=0$ and $\left(i_{2} f_{z}+i_{z} f_{2}\right)^{2}=1-\left(\frac{q}{n_{\mathrm{e}} k_{0}}\right)^{2}$, whereas for extraordinary incident light and ordinary scattered light (Fig. 4, panel b, right) one has $i_{1}=i_{2}=f_{1}=f_{z}=0$, which leads to $\left(i_{1} f_{z}+i_{z} f_{1}\right)^{2}=0$ and 


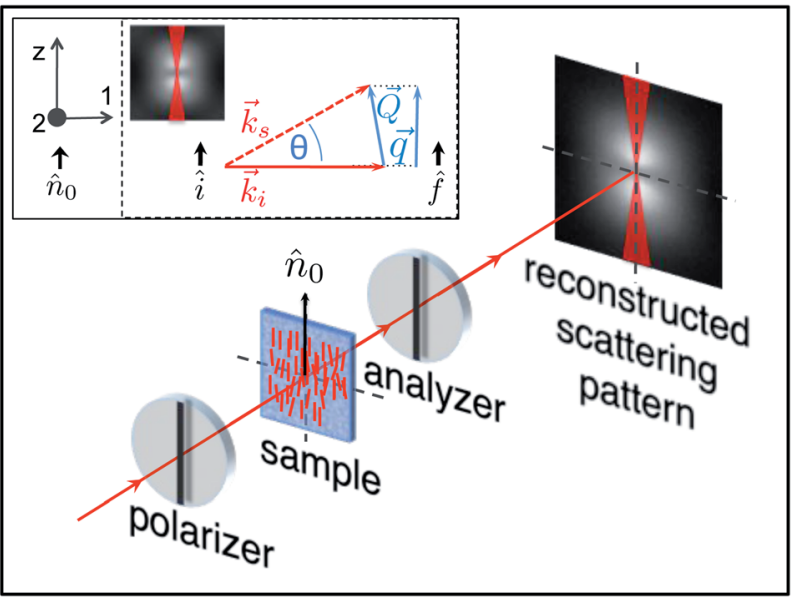

Fig. 5 Sketch of a pDDM experiment in the P2 geometry. Unpolarised light crosses the polariser and interacts with the nematic sample, whose director is parallel to the polariser axis. The analyser is oriented parallel to the polariser. In the inset we draw the reference system used for mode decomposition (left) and the scattering diagram (right) describing elastic scattering processes with $\vec{q} \| \hat{n}_{0}$.

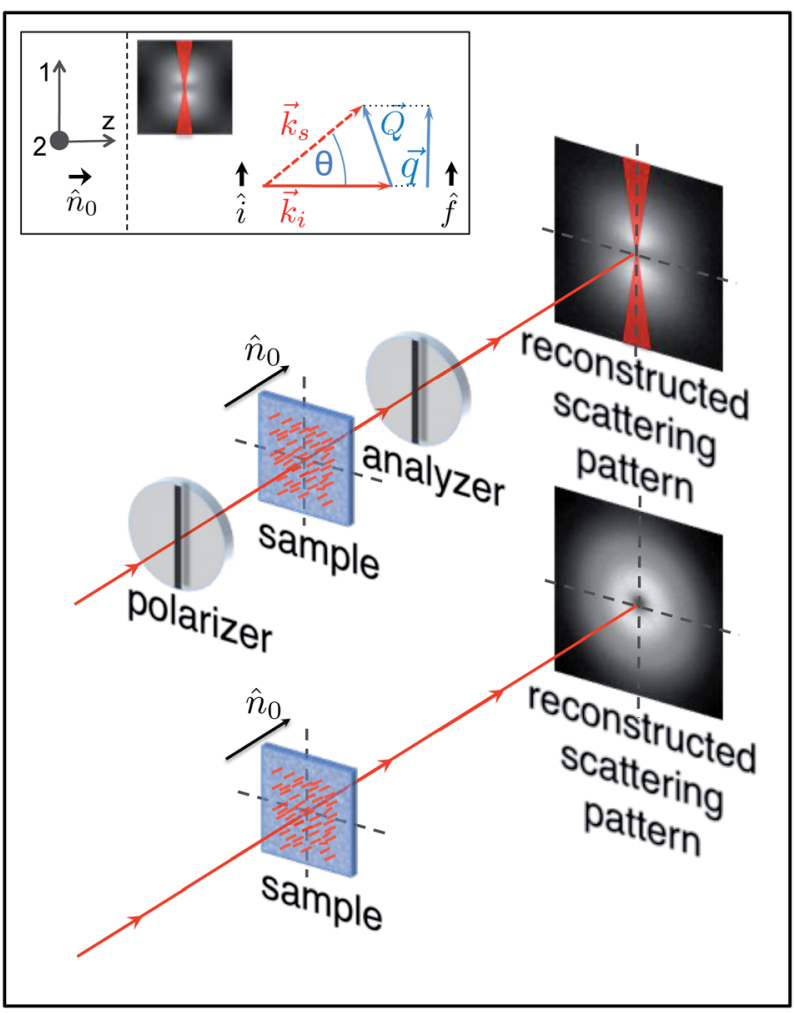

Fig. 6 Sketch of a pDDM experiment in the H1 geometry. In the configuration shown in the upper part of the main figure, unpolarised light crosses the polariser and interacts with the nematic sample, whose director is parallel to the optical axis. The analyser is oriented parallel to the polariser. In the configuration shown in the lower part of the figure all the polarising elements are removed. In the inset we draw the reference system used for mode decomposition (left) and the scattering diagram (right) describing elastic scattering processes with $\vec{q} \perp \hat{n}_{0}$.

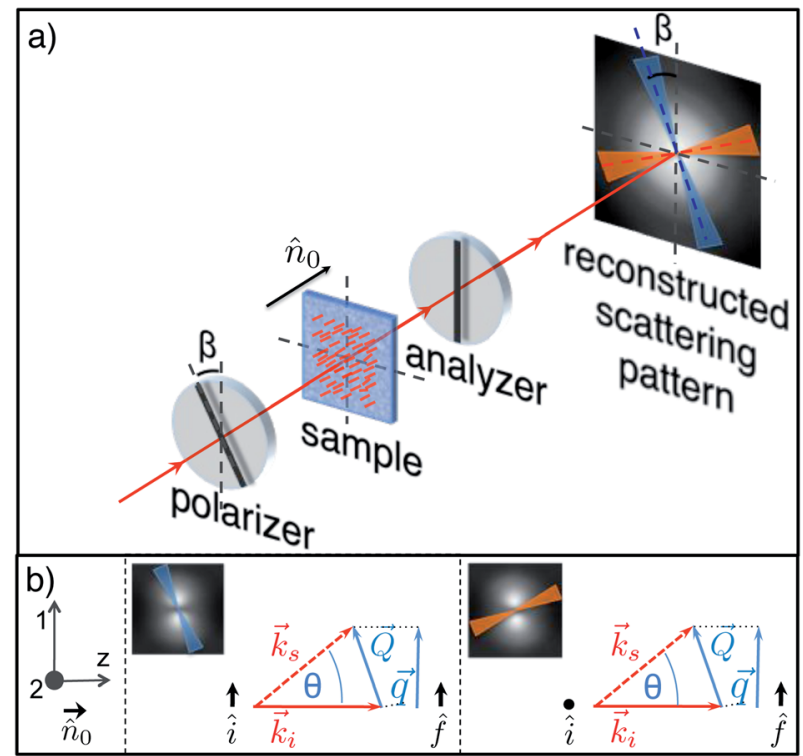

Fig. 7 (a) Sketch of a pDDM experiment in the $\mathrm{H} 2$ geometry. Unpolarised light crosses the polariser and interacts with the nematic sample, whose director is parallel to the optical axis. The analyser is oriented at $\beta=30^{\circ}$ with respect to the polariser. If the scattering pattern is analysed along the direction parallel (blue online) to the polariser the contribution of depolarised scattering is negligible and only polarised scattering is of interest. If the scattering pattern is analysed along the direction perpendicular (red online) to the polariser the only relevant contribution comes from depolarised scattering. (b) Reference system used for mode decomposition (left), the scattering diagram (centre) describing elastic scattering processes within the plane of the analyser and the scattering diagram (left) describing elastic scattering process within the plane perpendicular to the analyser.

$\left(i_{2} f_{z}+i_{z} f_{2}\right)^{2}=1$. Considering that $Q_{\|}=q$ and $Q_{\perp} \simeq \Delta q$, we have the following expression for the relaxation rate of mode 2 :

$$
\Gamma_{2}(q)=\frac{K_{22} \Delta q^{2}+K_{33} q^{2}}{\gamma_{1}-\frac{\alpha_{2}^{2} q^{2}}{\Delta q^{2} \eta_{a}+q^{2} \eta_{c}}}
$$

which can be expanded for $q \ll \Delta q \sqrt{\eta_{a} / \eta_{c}}$ to give

$$
\Gamma_{2}(q) \simeq \frac{K_{22}}{\gamma_{1}} \Delta q^{2}+\frac{K_{33}}{\gamma_{1}}\left(1+\frac{K_{22} \alpha_{2}^{2}}{K_{33} \gamma_{1} \eta_{a}}\right) q^{2}
$$

The rate $\Gamma_{2}(q)$ has a rather complex dependence on $q$. The intercept for $q \rightarrow 0$ provides the twist viscoelastic ratio. The bend ratio can be extracted only for large $q\left(q \gg \Delta q \sqrt{\eta_{a} / \eta_{c}}\right)$ where

$$
\Gamma_{2}(q) \simeq \frac{K_{33}}{\eta_{\text {bend }}} q^{2}
$$

It appears that, in the absence of prior information about the value of $\sqrt{\eta_{a} / \eta_{c}}$, it is not easy to know a priori whether the bend viscoelastic ratio in eqn (19) is experimentally accessible or one rather accesses the more complex combination in eqn (18).

- for $\vec{q}$ perpendicular to $\hat{n}_{0}$ the reference system is depicted on the left side of panel c in Fig. 4. Both for extraordinary 
incident light and ordinary scattered light (Fig. 4, panel c, centre) and ordinary incident light and extraordinary scattered light (Fig. 4, panel c, right) one has $\left(i_{1} f_{z}+i_{z} f_{1}\right)^{2}=q^{2} /\left(\Delta q^{2}+q^{2}\right)$ and $\left(i_{2} f_{z}+i_{z} f_{2}\right)^{2}=q^{2} /\left(\Delta q^{2}+q^{2}\right)$, which implies that both modes show-up in the scattering intensity. In addition, we have $Q_{\|}=0$ and $Q_{\perp}=\sqrt{\Delta q^{2}+q^{2}}$, which leads to

$$
\begin{aligned}
& \Gamma_{1}(q)=\frac{K_{11}}{\eta_{\text {splay }}}\left(q^{2}+\Delta q^{2}\right) \\
& \Gamma_{2}(q)=\frac{K_{22}}{\eta_{\text {twist }}}\left(q^{2}+\Delta q^{2}\right)
\end{aligned}
$$

for the relaxation rates of the two modes. For $q \ll\left(K_{11} / K_{22}\right) \Delta q$ the scattering from mode 2 dominates over mode 1 and the splay and twist contributions can be easily separated via doubleexponential fitting of the structure function by using eqn (16) or, when this is not possible, by inspecting the low- $q$ and the high- $q$ limits of the system dynamics, where a single exponential behaviour is a good approximation. It has to be mentioned that for low- $q$ the scattering is due to twist fluctuations as expected also from the fact that refractive index modulations with $\vec{q} \simeq 0$ are characterised by $\vec{Q}$ parallel to the $z$-axis. Modulations of this kind can only be originated by twist deformation of the LC director, which thereby act as Bragg diffraction grating with transmission coefficient peaked around $Q \simeq \Delta q$.

Planar alignment - geometry P2. In this configuration the polariser, the analyser and the director are all parallel to each other (Fig. 5). In contrast with the previous case, scattering is elastic here and $Q \simeq \Delta q$. Along the direction described by the bow-tie region in Fig. 5 (inset) the dynamics is due to mode 1 and in particular to bend fluctuations of the director. Indeed, $\left(i_{1} f_{z}+i_{z} f_{1}\right)^{2}=q^{2} /\left(n_{e} k_{0}\right)^{2}$ and $\left(i_{2} f_{z}+i_{z} f_{2}\right)^{2}=0$ and the relaxation rate is given by

$$
\Gamma_{1}(q)=\frac{K_{33}}{\eta_{\text {bend }}} q^{2}
$$

which, at variance with geometry $\mathrm{P} 1$, allows for an unambiguous determination of the bend viscoelastic ratio. In practice, a combination of P1 and P2 experiments allows always a full characterisation of the splay, twist and bend viscoelastic ratios, even though for some samples $\mathrm{P} 2$ experiments could prove to be unnecessary. For this geometry, a detailed theoretical treatment of the deep Fresnel diffraction from periodic modulations of the nematic LC was presented recently. ${ }^{\mathbf{1 6}}$ In particular, we can make use of the expression for the intensity $I$ given in eqn (48) of ref. 16 to write:

$$
A(q)=2 I_{0}^{2}(\beta q)^{2}\left|\delta \tilde{n}_{1}(Q)\right|^{2} \cos ^{2}\left(\frac{q^{2}}{2 k_{\mathrm{o}}} z^{\prime}\right)
$$

where $\beta=1-\left(m_{\mathrm{o}} / m_{\mathrm{e}}\right)^{2}, z^{\prime}$ is the observation distance and $\left|\delta \tilde{n}_{1}(Q)\right|^{2}$ is the $3 \mathrm{D}$ power spectrum of the director fluctuations within the scattering plane (mode 1 ). For $q \gg \pi / h$ (about 0.06 $\mu \mathrm{m}^{-1}$ for a sample thickness $h=50 \mu \mathrm{m}$ ) one can safely assume that $Q \simeq q$ and application of the equipartition theorem to eqn (23) gives the simple result

$$
A(q)=2 I_{0}{ }^{2} V \beta^{2} \frac{k_{\mathrm{B}} T}{K_{33}}
$$

where $V=L^{2} h$ is the probed sample volume, and $L$ is the length of the image side. Finally, even though the analysis in ref. 16 does not account for the effects of limited coherence of the light source and of the transfer function of the objective, such effects can be safely neglected at small enough $q,{ }^{9}$ which enables us to make use of eqn (24) to extract the bend elastic constant $K_{33}$ from our pDDM images acquired in the P2 geometry. We note here that in contrast to the case of non-absorbing colloidal particles, periodic modulations of the director in the P2 geometry do not behave as a simple phase grating, as it can be appreciated from eqn (23) that contains a $\cos ^{2}$ term instead of the usual $\sin ^{2}$ term. ${ }^{9}$ Quite interestingly, this shows that the birefringence of LC brings in additional ingredients to the problem of deep Fresnel scattering and in turn to the description of Differential Dynamic microscopy experiments. While these ingredients do not affect the determination of the LC dynamics, they need to be carefully accounted for when static scattering information is of interest.

Homeotropic alignment - geometry H1. If the sample is placed between parallel polarisers (Fig. 6) the intense, linearly polarised transmitted beam interferes only with scattered light with the same polarisation. Splay fluctuations with wave vector $\vec{Q} \simeq \vec{q}$ parallel to the polarising elements behave as a diffraction grating with wavelength $2 \pi / q$ and in fact, only polarised scattering due to splay fluctuations is probed. Indeed, one has for the two modes $\left(i_{1} f_{z}+i_{z} f_{1}\right)^{2}=q^{2} /\left(n_{\mathrm{o}} k_{0}\right)^{2}$ and $\left(i_{2} f_{z}+i_{z} f_{2}\right)^{2}=0$, with $\vec{Q} \simeq \vec{Q}_{\perp}$. The relaxation rate of such fluctuations is thus given by

$$
\Gamma_{1}(q)=\frac{K_{11}}{\eta_{\text {splay }}} q^{2}
$$

Interestingly, the removal of both polarising elements leaves the situation unchanged. Indeed, unpolarised light is the incoherent sum of different polarisation states but for each one of them only light produced by scattering processes that maintain the original polarisation can interfere with the transmitted beam. This leads to the advantage that the scattering pattern becomes azimuthally symmetric, and that in turn azimuthal averaging of the data can be performed to increase the statistical accuracy of the results (Fig. 6).

Homeotropic alignment - geometry H2. In this geometry, the polariser is not perpendicular to the analyser but it is rotated by an angle $\beta$ (for instance, for the experiments reported here we used $\beta=30^{\circ}$ ) with respect to that condition (Fig. 7). This configuration guarantees the fulfilment of the heterodyne condition and the validity of eqn (10). With respect to H1 geometry, here we have an additional depolarised scattering contribution. Indeed, similar to geometry P1, the incident light can be considered as the sum of two components, one with polarisation parallel to the polariser and the other one perpendicular to it. Because of the presence of the analyser both components can now interfere with the transmitted beam.

- for director modulations with wave vector along the polariser direction, mode 1 is probed and, in analogy with geometry $\mathrm{H} 1$, splay-induced polarised scattering is observed. Indeed, one has $\left(i_{1} f_{z}+i_{z} f_{1}\right)^{2}=q^{2} /\left(n_{\mathrm{o}} k_{0}\right)^{2}$ and $\left(i_{2} f_{z}+i_{z} f_{2}\right)^{2}=0$, with $\vec{Q} \simeq \vec{Q}_{\perp}$. The relaxation rate of such fluctuations is 


$$
\Gamma_{1}(q)=\frac{K_{11}}{\eta_{\text {splay }}} q^{2}
$$

- in the direction perpendicular to the polariser, depolarised scattering is sensitive to mode 2 and $\left(i_{1} f_{z}+i_{z} f_{1}\right)^{2}=0,\left(i_{2} f_{z}+i_{z} f_{2}\right)^{2}$ $=q^{2} /\left(n_{\mathrm{o}} k_{0}\right)^{2}$ with $\vec{Q} \simeq \vec{Q}_{\perp}$. Twist fluctuations with wave vector $\vec{Q}$ $\simeq \vec{q}$ act thus as a diffraction grating with wavelength $2 \pi / q$ and the relaxation rate of such fluctuations is thus given by

$$
\Gamma_{2}(q)=\frac{K_{22}}{\eta_{\text {twist }}} q^{2} .
$$

It appears that a combination of P1 and P2 measurements always allows for a complete characterisation of the LC viscoelasticity, with geometry P1 being sufficient under some circumstances. By contrast, measurements on a homeotropically aligned LC can not provide access to the bend viscoelastic ratio.

\section{Experimental}

For our experiments we use the thermotropic liquid crystal 4-hexyl-4'-cynanobiphenyl (6CB, Sigma-Aldrich), whose nematic phase is in the temperature range $14.5-28.8^{\circ} \mathrm{C}$. We consider two samples that are confined in cells made of optical-quality glass windows and that differ only in the alignment of the LC molecules at the cell surface. To that purpose the cell surfaces in contact with the LC sample are treated so as to favour either parallel (planar or $\mathrm{P}$ ) or perpendicular (homeotropic or $\mathrm{H}$ ) alignment. $\mathrm{H}$ alignment is obtained by depositing a layer of polyimide (polymer 0626 from Nissan Chemistry Industries) with spin coating (3000 rpm for 2 minutes), whereas for $\mathrm{P}$ alignment, spin coating of a $0.5 \%$ water solution of polyvinylalcohol (3000 rpm for 2 minutes) is followed by rubbing. By using Mylar ${ }^{\circledR}$ spacers, the cell thickness is set to $h=50 \pm 3 \mu \mathrm{m}$, as checked with optical Fabry-Perot interferometry in reflection. The cell is then filled with 6CB in the isotropic phase at $T=$ $40{ }^{\circ} \mathrm{C}$ and sealed with epoxy glue. The sample is subsequently cooled at a low rate from the isotropic to the nematic phase and eventually kept for at least one hour at $10^{\circ} \mathrm{C}$ (or $12{ }^{\circ} \mathrm{C}$ for some of the experiments) below the nematic-isotropic transition temperature $T_{\mathrm{NI}}=28.8{ }^{\circ} \mathrm{C}$. Measurements are performed at different temperatures in the nematic phase after careful thermalisation of the sample. Thermalisation takes place inside an INSTEC-HCS301I hot stage mounted on a NIKON Eclipse Ti-U microscope, which is also used for the sample observation. At each temperature, images of the fluctuations are acquired with a fast camera (IDT M3), with the image pixel size corresponding to $d_{\text {pix }}=1.2 \mu \mathrm{m}$ in real space (magnification $10 \times$ ). A typical dataset consists of a sequence of 8000 images, acquired with a sampling rate of 2500 images per $\mathrm{s}$ and an exposure time of $400 \mu \mathrm{s}$. The total measurement time is thus $3.2 \mathrm{~s}$ for each temperature and geometry. The acquired images are stored on a disk for subsequent pDDM analysis, which is performed by using MATLAB ${ }^{\circledR}$. In contrast with bright field DDM experiments, the 2D structure functions of LC do not bear in general
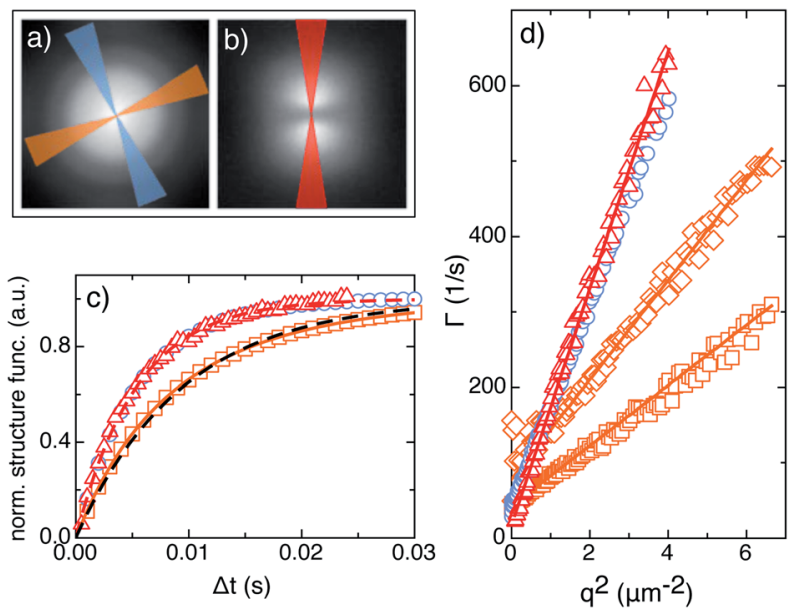

Fig. 8 (a) Reconstructed scattering pattern $A\left(q_{x}, q_{y}\right)$ for the P1 geometry. The two shaded bow-tie areas, centred, respectively, around a direction parallel (blue) and perpendicular (orange) to the nematic director $\hat{n}_{0}$, indicate the regions where the azimuthal averages are performed. (b) Reconstructed scattering pattern for the P2 geometry. The red shaded area, oriented along the direction of the nematic director, indicates the region used for the azimuthal average. (c) Normalised structure functions for the P1 and P2 geometries at $q=$ $1.0 \mu^{-1}$. Blue circles (orange squares) correspond to $\vec{q}$ parallel (perpendicular) to $\hat{n}_{0}$ in the P1 geometry. Red triangles correspond to $\vec{q}$ parallel to $\hat{n}_{0}$ in the P2 geometry. The dashed line is the best fitting single exponential curve for $\vec{q} \| \hat{n}_{0}$, while the continuous blue line is the best fitting double exponential curve for $\vec{q} \perp \hat{n}_{0}$. (d) $q$-dependent relaxation rates $\Gamma$ obtained with the $\mathrm{P} 1$ (circles, squares and diamonds) and the P2 (triangles) geometries.

an azimuthal symmetry, as it can be appreciated in Fig. $4-7$. As a consequence, instead of azimuthally averaging the data with the same $q$ but different $\left(q_{x}, q_{y}\right)$ over $2 \pi$, we limit the angular size of the region over which the averaging is performed by using bowtie shaped regions, as shown in Fig. 8 (panels a and b) and 11, for the planar and homeotropic samples, respectively.

\section{Results and discussion}

\section{Planar alignment}

At each temperature, data for planar samples are acquired first in the P1 geometry (polariser $\perp$ analyser) and immediately after in the P2 geometry (polariser || analyser) by rotating the sample and the polariser. According to the expectations from theory (Section 3), the reconstructed scattering patterns $A\left(q_{x}\right.$, $q_{y}$ ) for the P1 (Fig. 8a) and P2 (Fig. 8b) geometries have quite different symmetry properties. As far as the pDDM analysis is concerned, for the P1 geometry, the two shaded bow-tie areas (angular width $\pi / 32$ ) in Fig. 8a indicate the two regions used for azimuthal averaging, one (blue) with the scattering wave vector $\vec{q}$ parallel to the director $\hat{n}_{0}$, whereas the other (orange) with $\vec{q}$ perpendicular to $\hat{n}_{0}$. It can be noticed that the two highlighted regions are rotated by $\pi / 8$ with respect to the image axes as a consequence of the fact that in this geometry the director forms an angle $\pi / 8$ with the polariser (Fig. $4 \mathrm{a}$ ). By contrast, the scattering pattern for the $\mathrm{P} 2$ geometry is almost concentrated in a direction parallel to the director (Fig. 8b), as scattering vanishes 
perpendicularly to it. The region used for the pDDM analysis is highlighted in blue and has an angular width of $\pi / 16$. In Fig. $8 \mathrm{c}$ we plot three structure functions measured at $T-T_{\mathrm{NI}}=0.2{ }^{\circ} \mathrm{C}$ and for $q=1.0 \mu \mathrm{m}^{-1}$ in the $\mathrm{P} 1$ and in the $\mathrm{P} 2$ geometries. It appears that relaxation of the director fluctuations when $\vec{q}$ is perpendicular to $\hat{n}_{0}$ (orange squares in Fig. $8 \mathrm{c}$ for the P1 geometry) are slower than fluctuations in the parallel direction (blue circles for the P1 geometry and red triangles for the P2 geometry in Fig. 8c). According to the theoretical expectation, the P2 geometry is the easiest case to analyse. Indeed, a single mode (mode 1) of pure bend is probed with a rate given by eqn (22). Fitting the structure function with a single exponential curve (red dashed line in Fig. 8c) provides the rate $\Gamma_{1}$, which is plotted as a function of $q^{2}$ in Fig. 8d (red triangles). For each temperature, the obtained results are well fitted with a linear function, from which the bend ratio $K_{33} / \eta_{\text {bend }}$ can be extracted. Results for different temperatures are reported in Fig. 9a (full red circles) and are in excellent agreement with previous experiments on the same sample with DDLS. ${ }^{28}$ The situation is more complex for the P1 geometry. For $\vec{q}$ parallel to $\hat{n}_{0}$, scattering is originated only from mode 2 and thus the dynamics is again well described by a single exponential function (blue circles in Fig. 8c). The rate $\Gamma_{2}$ extracted from the single exponential fitting is plotted in Fig. 8d (blue circles) as a function of $q^{2}$. In principle, these data should be fitted with eqn (17). However, this fit becomes very challenging because of the large number of fitting parameters and the limited $q$-range of the experiments. In addition, the possible use of the simpler expression in eqn (19) to extract the bend ratio is based on prior knowledge of $\eta_{a}, \eta_{c}$ and $\Delta q$ to ensure that the condition $q \gg \Delta q \sqrt{\eta_{a} / \eta_{c}}$ is met. Literature data for $\eta_{a}$ and $\eta_{c}$ relative to our sample could not be retrieved. However, data for $5 \mathrm{CB}^{26}$ and $\mathrm{MBBA}^{27}$ in a $T-T_{\mathrm{NI}}$ range similar to the one explored here show that $\sqrt{\eta_{a} / \eta_{c}}$ remains in the range 1-1.7. Based on this estimate, we can expect the condition $q \gg \Delta q \sqrt{\eta_{a} / \eta_{c}}$ to be met only in a narrow range close to the critical temperature $T_{\mathrm{NI}}$, where in principle it should be possible to extract the bend viscoelastic ratio. In fact, even quite close to $T_{\mathrm{NI}}$, the approximation of eqn (17) with eqn (19) is still not fully satisfactory, as it can be appreciated in Fig. 8d, by comparing the rates obtained in this condition (blue circles) with the ones obtained in the P2 geometry (red triangles). For $\vec{q}$ perpendicular to $\hat{n}_{0}$, the structure function is the sum of two exponential functions due to the superposition of modes 1 and 2 (eqn (16)). A double exponential fit (continuous line) thus provides the corresponding relaxation rates $\Gamma_{1}(q)$ and $\Gamma_{2}(q)$ (diamonds and squares in Fig. 8d, respectively). For small $q$, where scattering from mode 2 dominates, data for $\Gamma_{1}(q)$ appear quite noisy, as the small amplitude of mode 1 translates into a large uncertainty in determining $\Gamma_{1}(q)$. Nevertheless, data for both modes can be well fitted to eqn (20) and (21) to extract the splay and twist viscoelastic ratios, respectively and $\Delta q$. The results for different temperatures are shown as orange down-triangles in Fig. 9b and c, respectively. Again the agreement with previous experiments in ref. 28 is very good.

As a by-product of the analysis in the P1 geometry, the birefringence $\Delta m$ can be obtained from the experimentally
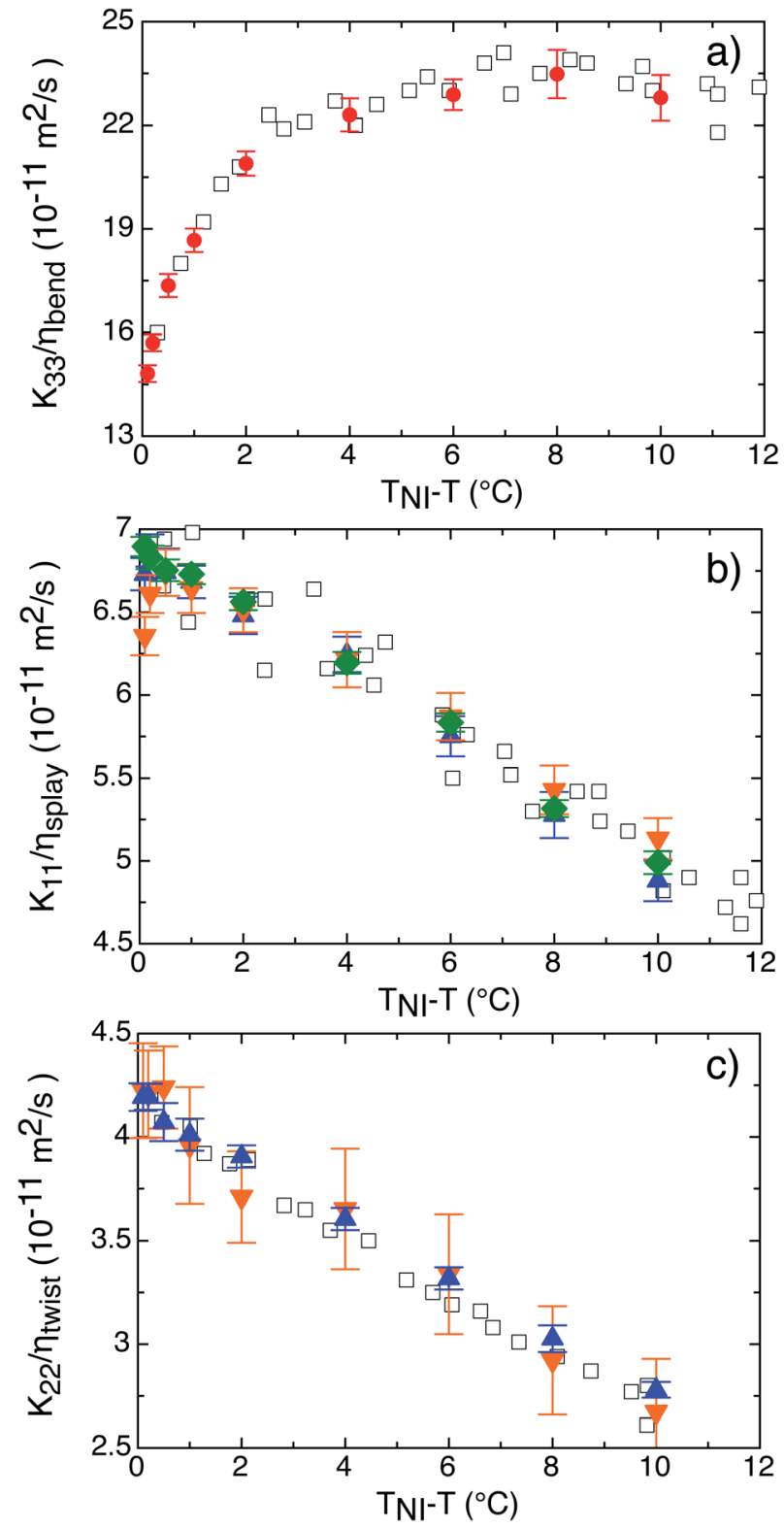

Fig. 9 Viscoelastic ratios of $6 \mathrm{CB}$ measured as a function of the temperature difference from the transition temperature $T_{\mathrm{N} I}$. Full symbols are obtained with pDDM in different geometries (P1 geometry - red circles, P2 geometry - orange down-triangles, $\mathrm{H} 1$ geometry green diamonds, $\mathrm{H} 2$ geometry - blue up-triangles). Empty symbols are literature data (from ref. 28) obtained with DDLS.

determined $\Delta q$, by using the relationship $\Delta q=k_{0} \Delta m=\left(2 \pi / \lambda_{0}\right) \Delta m$, where we used $\lambda_{0}=580 \mathrm{~nm}$ for the peak wavelength of our light source. ${ }^{9}$ Results obtained in this way for $\Delta m$ at different temperatures are reported in Fig. 10a (black circles) together with the literature data obtained with traditional refractometry (red squares). ${ }^{27}$

Finally, by making use of eqn (24) it is also possible to calculate the bend elastic constant from the amplitude $A(q)$ of the structure functions for $\vec{q}$ parallel to $\hat{n}_{0}$ obtained for each temperature in the $\mathrm{P} 2$ geometry. A reliable estimate of the low- $q$ limit $A_{0}$ of the amplitude is obtained as the average of $A(q)$ over the interval $[0.39,0.49] \mu \mathrm{m}^{-1}$, where $A(q)$ is essentially flat. 

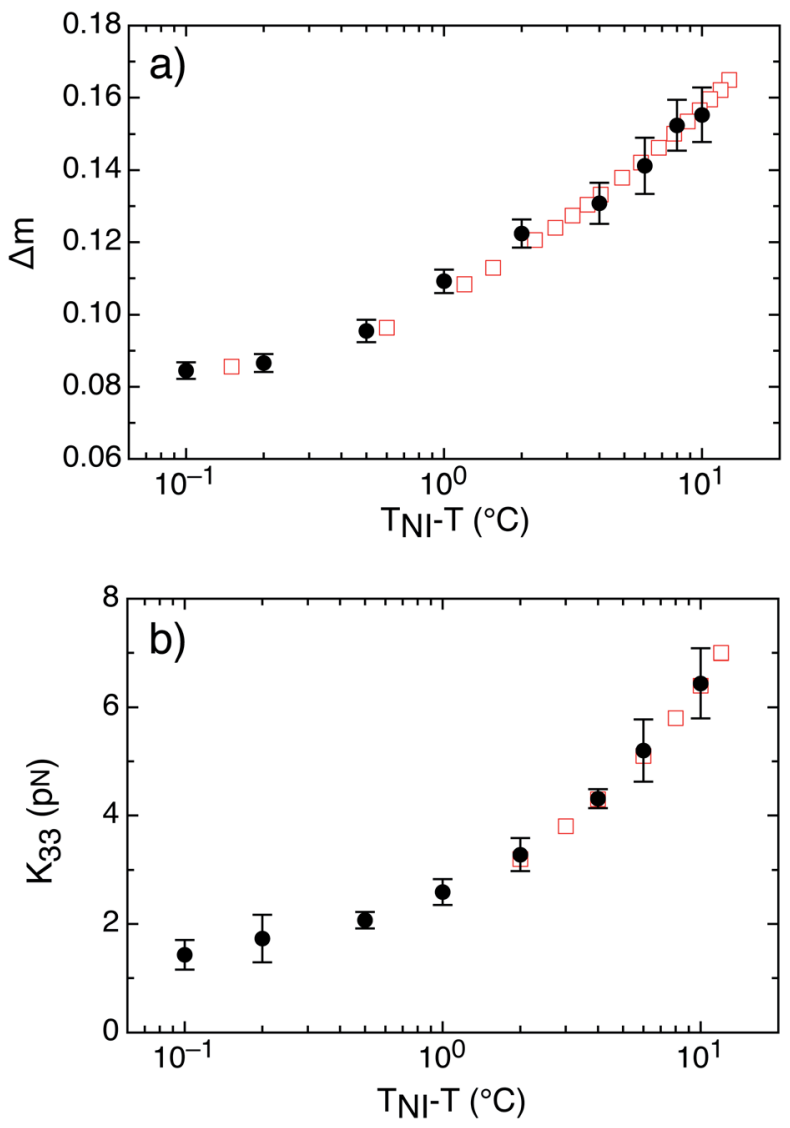

Fig. 10 Experimentally determined birefringence (panel a) and bend elastic constant (panel b) of $6 \mathrm{CB}$ as a function of the temperature difference from the transition temperature $T_{\mathrm{N} \text {. }}$. Black circles are data obtained with pDDM. Red squares are literature data from ref. 27.

According to eqn (24), the bend elastic constant $K_{33}$ is estimated as $K_{33}=2 I_{0}{ }^{2} V \beta^{2} k_{\mathrm{B}} T / A_{0}$. As shown in Fig. 10, the obtained values compare remarkably well with the literature data obtained by light scattering. ${ }^{27}$ Most remarkably, we are able to extend the existing data-range by almost a decade with pDDM approaching further towards $T_{\mathrm{NI}}$. This is because pDDM is less sensitive to multiple scattering as compared to traditional light scattering methods. We note that in principle the good result obtained for $K_{33}$ could be extended also to the other two elastic constants, provided that some expressions analogous to eqn (24) are available for the corresponding geometries. Given the complexity of the calculations involved, the derivation of such expressions is well beyond the purpose of the present article, but it should be a priority for further developing the full potential of the method.

\section{Homeotropic alignment}

At each temperature we have performed measurements first in the $\mathrm{H} 1$ geometry (no polarisers, Fig. 11b) and successively in the $\mathrm{H} 2$ geometry (polariser and analyser mutually oriented at $60^{\circ}$ ). In $\mathrm{H} 1$ geometry (mode 1 ) the splay ratio can be obtained by taking advantage of the azimuthal symmetry of the scattering pattern (Fig. 11b) whereas both splay and twist ratios can be

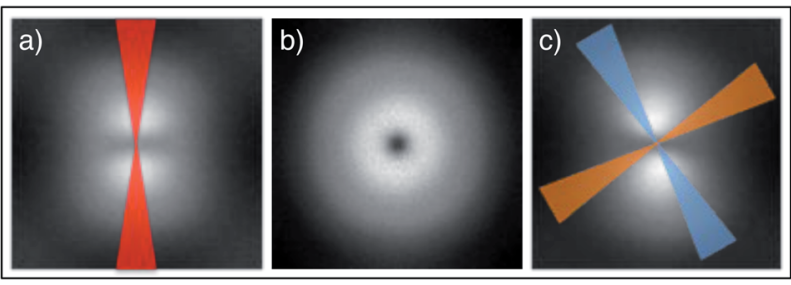

Fig. 11 (a) Reconstructed scattering pattern for the $\mathrm{H} 1$ geometry (parallel polarisers). The red shaded bow-tie area, oriented along the direction of the axis of the polarising elements, indicates the region where the azimuthal average is performed. (b) Reconstructed scattering pattern for the $\mathrm{H1}$ geometry (no polarisers). (c) Reconstructed scattering pattern for the $\mathrm{H} 2$ geometry. The two shaded bow-tie areas are oriented, respectively, along a direction parallel (blue) and perpendicular (orange) to the axis of the polariser and indicate the regions where the azimuthal averages are performed.

extracted in $\mathrm{H} 2$ geometry (mode 1 and 2 ) by analysing the dynamics in two perpendicular directions in the $q$-space.

The results are reported in Fig. $9 \mathrm{~b}$ and $\mathrm{c}$, where green diamonds are obtained in the $\mathrm{H} 1$ geometry, whereas blue uptriangles in the $\mathrm{H} 2$ geometry. All the results are in excellent agreement with both the literature data and the measurements are performed in the P1 geometry. We note that the quality of the results obtained with the $\mathrm{H} 1$ geometry, where azimuthal averaging of the structure functions over $2 \pi$ was performed, is appreciably higher than that obtained in other geometries. This also shows that polarising elements are not always needed for the characterisation of the viscoelastic behaviour of LC.

\section{Heterogeneous planar alignment}

To explore the novel possibilities offered by this imaging-based scattering method, a last set of experiments was performed with a sample of 6CB confined in a cell whose glass surfaces were kept untreated, to obtain a heterogeneous planar alignment that occurs with many samples for which alignment procedures are not available or known, such as for instance biological LC formers. ${ }^{17}$ The alignment of the LC molecules at the surfaces is spontaneously planar but does not remain uniform across the cell width. This alignment is also known in the literature as random planar alignment. Images acquired between crossed polarisers under this condition have the typical Schlieren texture appearance (Fig. 12a). However, it is still possible to identify small domains inside which the planar alignment is approximately uniform even though different domains are characterised by different orientations of the director.

To investigate the amount of quantitative information that can be extracted in this condition we have prepared a sample of 6CB with heterogeneous planar alignment that was characterised at the fixed temperature $T=22.7 \pm 0.2{ }^{\circ} \mathrm{C}$. Two movies of the same region (8000 images with $512 \times 512$ resolution) were acquired, respectively, at 800 and 1000 frames per second. The first movie was taken with the sample placed between perpendicular polarisers (Fig. 12a), while for the second the polarisers were kept parallel (Fig. 12b). Such double acquisition enabled us to identify proper sub-regions for the pDDM analysis as 


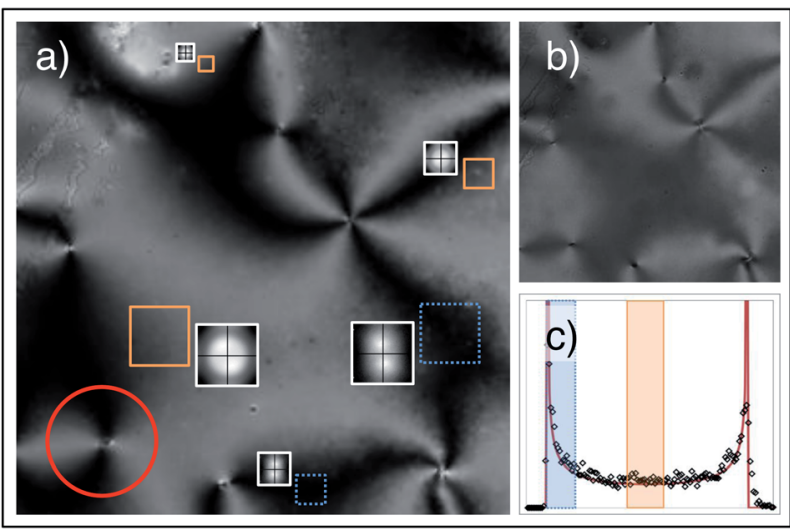

Fig. 12 Portion of a heterogeneous planar sample of 6CB observed between crossed (a) and parallel (b) polarisers. (c) Intensity histogram (symbols) of the area enclosed in the red circle in panel (a), centred around a point-like defect. The continuous line is the best fitting curve of the form: $P(I)=(1 / \pi)\left(I-I_{\min }\right)^{-1 / 2}\left(I_{\max }-I\right)^{-1 / 2}$, expected from theory. The intensity range outlined in blue (orange) with dotted (continuous) contour corresponds to regions where the nematic director is approximately parallel (tilted by $\pi / 8+n \pi / 4$, with $n$ integer) with respect to the polarising element. In panel (a) some representative regions of interest are shown, corresponding to these orientations; dotted blue squares were analysed with pDDM in the P1 geometry, while orange squares in the P2 geometry. The corresponding reconstructed scattering patterns are also shown close to each region of interest.

follows. A point-like defect and the corresponding region of interest such as the one outlined with a red circle in Fig. 12a is chosen under the assumption that the orientation of the director around it is uniformly distributed. The image intensity histogram of the region of interest similar to the one in Fig. 12a is calculated and fitted with the theoretical expression: $P(I)=$ $(1 / \pi)\left(I-I_{\min }\right)^{-1 / 2}\left(I_{\max }-I\right)^{-1 / 2}$ derived under the hypothesis of uniform distribution of the director orientation to obtain a correspondence between intensity levels in the image and director angle (modulo $\pi / 4$ ). Once such correspondence is obtained (Fig. 12a) the analysis proceeds differently according to the viscoelastic ratio of interest.

Splay and twist. For the determination of the splay and twist viscoelastic ratios we select an intensity range at the centre of the histogram and determine the corresponding regions in the image, where the director is oriented at $\pi / 8+n \pi / 4$ (n integer), with respect to the polarising elements. Inside these regions small square regions of interest (ROI) $(64 \times 64$ or $32 \times 32$ or 16 $\times 16$ pixels) are selected and a pDDM analysis is run in parallel for all of them. The actual orientation of the director within each ROI can be easily determined by exploiting the asymmetry displayed by the 2D structure function, as shown in Fig. 11a. The structure functions with $\vec{q}$ perpendicular to $\hat{n}_{0}$ are analysed and fitted with a single exponential, since the limited size of the square regions of interests prevents a double exponential fit. Nevertheless, the twist and splay viscoelastic ratios can be extracted from the $\Gamma(q)$ data in Fig. 13. The results for the two ratios $\left(K_{11} / \eta_{\text {splay }}=(5.9 \pm 0.3) \times 10^{-11} \mathrm{~m}^{2} \mathrm{~s}^{-1}, K_{22} / \eta_{\text {twist }}=(2.6 \pm\right.$ $0.6) \times 10^{-11} \mathrm{~m}^{2} \mathrm{~s}^{-1}$ ) are in fair agreement with those obtained with uniformly aligned samples, reported in Fig. 9.

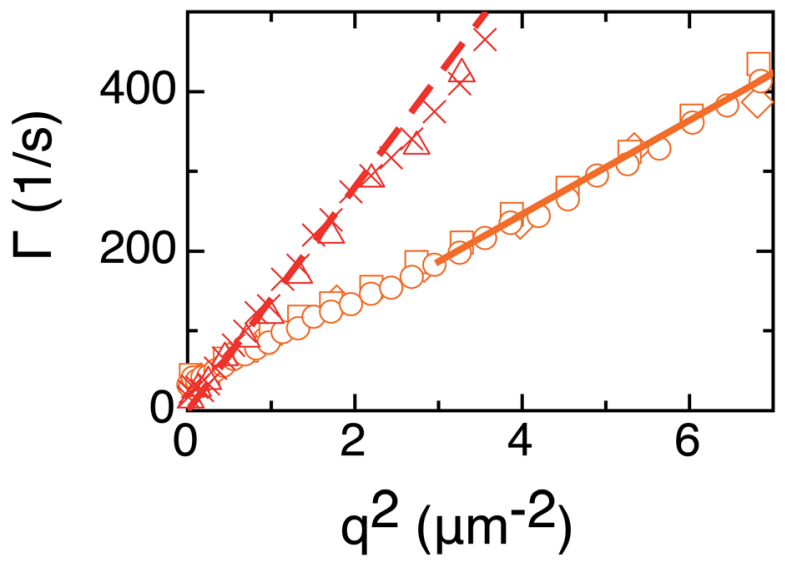

Fig. $13 q$-dependent relaxation rates $\Gamma$ obtained for a heterogeneous planar sample of $6 \mathrm{CB}$ at $T=22.7 \pm 0.2{ }^{\circ} \mathrm{C}$. Different symbols correspond to the different regions of interest in the sample, as shown in Fig. 12. Circles, squares and diamonds correspond to regions of interest equivalent to a P1 geometry experiment. Crosses and empty triangles are equivalent to a P2 geometry experiment. The shown fitting lines allow extraction of the bend (dashed line) and the splay (continuous line) ratios, whereas the extrapolated $q=0$ limit of the P1 data provides an estimate of the twist ratio.

Bend. An intensity range in the lower part of the image histogram is selected and the corresponding regions in the image are determined, where the director is parallel or perpendicular to the polarising elements. Since we are interested only in the regions with the director parallel to the polarisers, we focus on the regions where the intensity is larger and select small square regions of interest $(32 \times 32$ or $16 \times 16$ pixels) for the pDDM analysis. The analysis proceeds in each region of interest by using the same method used for the P2 geometry. The bend ratio obtained from the slope of the $\Gamma(q)$ curves in Fig. 13 is $K_{33} / \eta_{\text {bend }}=(1.3 \pm 0.3) \times 10^{-10} \mathrm{~m}^{2} \mathrm{~s}^{-1}$, which is about $70 \%$ smaller than the result obtained with homogeneously planar samples. This discrepancy can be attributed to a large, unknown pre-tilt angle at the surfaces, as independently verified with experiments performed on homogenous planar samples confined between glass plates rubbed in opposite directions, or to an unavoidable twist ${ }^{29}$ due to the possibly different orientation of the director on the two cell surfaces.

\section{Conclusions}

Our experiments demonstrate the versatile use of pDDM for the characterisation of the dynamics of liquid crystals and, in perspective, of other optically anisotropic fluids. It is worth stressing that the differential algorithm provides a very effective solution to the stray light problem, which makes DDLS measurements at small angles very challenging. Our method could thus be used as a robust analytical tool that would function in harsh environments as those typically found in production plants. More theoretical work will be needed to exploit the full potential of the method, not only for the characterisation of the three viscoelastic ratios but also for the corresponding elastic constant. 


\section{Acknowledgements}

We acknowledge funding by the Italian Ministry of Education and Research, "Futuro in Ricerca" Project ANISOFT (RBFR125H0M), from the CARIPLO grant 2008-2413 and from the Swiss National Science Foundation grant 200020-140908.

\section{References}

1 S. S. Choi, S. M. Morris, W. T. S. Huck and H. J. Coles, Soft Matter, 2009, 5, 354.

2 F. Serra, K. C. Vishnubhatla, M. Buscaglia, R. Cerbino, R. Osellame, G. Cerullo and T. Bellini, Soft Matter, 2011, 7, 10945.

3 Y. D. Liu and H. J. Choi, Soft Matter, 2012, 8, 11961.

4 S. M. Kelly and M. O'Neill, Liquid crystals for electro-optic applications, in Handbook of Advanced Electronic and Photonic Materials and Devices, ed. H. S. Nalwa, vol. 7, Liquid Crystals, Display and Laser Materials, 2001.

5 P. G. de Gennes and J. Prost, The Physics of Liquid Crystals, Oxford University Press, London, 2001.

6 H. F. Gleeson, in Handbook of Liquid Crystals, ed. D. Demus, J. Goodby, G. W. Gray and H.-W. Spiess, Wiley-VCH, Halle, 2008, pp. 699-718.

7 H. Orihara, A. Sakai and T. Nagaya, Mol. Cryst. Liq. Cryst., 2001, 366, 143.

8 R. Cerbino and V. Trappe, Phys. Rev. Lett., 2008, 100, 188102. 9 F. Giavazzi, D. Brogioli, V. Trappe, T. Bellini and R. Cerbino, Phys. Rev. E: Stat., Nonlinear, Soft Matter Phys., 2009, 80, 031403.

10 F. Ferri, A. D'Angelo, M. Lee, A. Lotti, M. C. Pigazzini, K. Singh and R. Cerbino, Eur. Phys. J.: Spec. Top., 2011, 199, 139.

11 L. G. Wilson, V. A. Martinez, J. Schwarz-Linek, J. Tailleur, G. Bryant, P. N. Pusey and W. C. K. Poon, Phys. Rev. Lett., 2011, 106, 018101.

12 V. A. Martinez, R. Besseling, O. A. Croze, J. Tailleur, M. Reufer, J. Schwarz-Linek, L. G. Wilson, M. A. Bees and W. C. K. Poon, Biophys. J., 2012, 103, 1637.
13 M. Reufer, V. A. Martinez, P. Schurtenberger and W. C. Poon, Langmuir, 2012, 28, 4618.

14 K. He, M. Spannuth, J. C. Conrad and R. Krishnamoorti, Soft Matter, 2012, 8, 11933.

15 P. J. Lu, F. Giavazzi, T. E. Angelini, E. Zaccarelli, F. Jargstorff, A. B. Schofield, J. N. Wilking, M. B. Romanowsky, D. A. Weitz and R. Cerbino, Phys. Rev. Lett., 2012, 108, 218103.

16 W. Pesch and A. Krekhov, Phys. Rev. E: Stat., Nonlinear, Soft Matter Phys., 2013, 87, 052504.

17 M. Nakata, G. Zanchetta, B. D. Chapman, C. D. Jones, J. O. Cross, R. Pindak, T. Bellini and N. A. Clark, Science, 2007, 318, 1276.

18 G. Zanchetta and R. Cerbino, J. Phys.: Condens. Matter, 2010, 22, 323102.

19 S. Chakraborty, J. T. Gleeson, A. Jakli and S. Sprunt, Soft Matter, 2013, 9, 1817.

20 A. S. Poulos, D. Constantin, P. Davidson, B. Pansu, E. Freyssingeas, A. Madsen and J. Chanac, J. Chem. Phys., 2012, 132, 091101.

21 P. Oswald and P. Pieranski, Nematic and Cholesteric Liquid Crystals, CRC Press, Boca Raton, 2005.

22 R. Cerbino and A. Vailati, Curr. Opin. Colloid Interface Sci., 2009, 14, 416.

23 F. Ferri, Rev. Sci. Instrum., 1997, 68, 2265.

24 L. Cipelletti and D. A. Weitz, Rev. Sci. Instrum., 1999, 70, 3214.

25 D. Brogioli, D. Salerno, V. Cassina, S. Sacanna, A. P. Philipse, F. Croccolo and F. Mantegazza, Opt. Express, 2009, 17, 1222.

26 C. P. Lapointe, Elastic Interactions and Manipulation of Wire-shaped Inclusions in Nematic Liquid Crystals, PhD thesis, The Johns Hopkins University, 2009.

27 Physical properties of liquid crystals: nematics, ed. D. A. Dunmur, A. Fukuda and G. R. Luckhurst, INSPEC, 2001.

28 J. Hirakata, G. Chen, T. Toyooka, S. Kawamoto, H. Takezoe and A. Fukuda, Jpn. J. Appl. Phys., 1986, 25, L607.

29 M. Buscaglia, G. Lombardo, L. Cavalli, R. Barberi and T. Bellini, Soft Matter, 2010, 6, 5434. 